\title{
Expression of the Mammalian Target of Rapamycin Pathway Markers in Lung Adenocarcinoma and Squamous Cell Carcinoma
}

\author{
Hyun-Soo Kim Gou Young Kim Sung-Jig Lim Youn Wha Kim \\ Department of Pathology, Kyung Hee University School of Medicine, Seoul, Republic of Korea
}

\section{Key Words}

Adenocarcinoma $\cdot$ Lung $\cdot \mathrm{mTOR} \cdot \mathrm{p} 70 \mathrm{S6} \mathrm{K} \cdot \mathrm{pAkt} \cdot \mathrm{PTEN} \cdot$

Rapamycin $\cdot$ Squamous cell carcinoma

\begin{abstract}
Objective: We investigated whether the expression of mammalian target of rapamycin (mTOR) pathway components is associated with clinicopathologic characteristics and patient outcome in lung adenocarcinoma (AC) and squamous cell carcinoma (SCC). Methods: We used immunohistochemistry to evaluate the expression of phosphorylated Akt (pAkt), mTOR, p70 ribosomal protein S6 kinase (p70S6K) and phosphatase and tensin homolog deleted on chromosome 10 (PTEN) in 91 cases of AC and 154 cases of SCC. Results: pAkt expression was positively correlated with the expression of mTOR $(p<0.001)$ and p70S6K $(p<0.001)$, and mTOR expression was positively correlated with p7056K expression $(p<$ $0.001)$. PTEN expression was inversely correlated with the expression of pAkt $(p=0.001)$, mTOR $(p<0.001)$ and p70S6K $(p=0.012)$. In addition, loss of PTEN expression, observed in $37.4 \%$ (34/91) of AC patients, was significantly associated with a higher histologic grade $(p=0.013)$, pathologic T stage $(p=0.016)$ and $N$ stage $(p<0.001)$ and advanced TNM stage $(p=0.001)$, as well as a shorter overall survival of AC patients
\end{abstract}

$(p=0.015)$. Conclusion: The high prevalence of PTEN loss and its association with aggressive tumor behavior and poor patient outcome in AC suggest that loss of PTEN expression is involved in $A C$ progression and serves as a prognostic marker for patients with AC.

Copyright $\odot 2012$ S. Karger AG, Basel

\section{Introduction}

Lung cancer is one of the most common causes of cancer death worldwide with consistently higher mortality than breast, colon and prostate cancers combined $[1,2]$. Non-small cell lung carcinoma (NSCLC) comprises about $80 \%$ of all diagnosed lung cancers. Despite recent advances in diagnosis and therapy, the prognosis for patients with NSCLC remains very poor with a 5-year cumulative survival rate of only $15 \%$. Improving the survival rate of patients with NSCLC will require a better understanding of tumor biology and the development of novel therapeutic strategies.

Mammalian target of rapamycin (mTOR) is an intracellular serine/threonine kinase that controls protein synthesis and cell cycle progression. As shown in figure $1, \mathrm{mTOR}$ is activated by phosphatidylinositol 3-kinase

\section{KARGER}

Fax +41613061234

E-Mail karger@karger.ch

www.karger.com
(C) 2012 S. Karger AG, Basel

$1015-2008 / 12 / 0792-0084 \$ 38.00 / 0$

Accessible online at:

www.karger.com/pat
Dr. Gou Young Kim

Department of Pathology, Kyung Hee University School of Medicine

1 Hoegi-dong, Dongdaemun-gu

Seoul 130-702 (Republic of Korea)

Tel. +82 2440 7551, E-Mail pathogen@medimail.co.kr 
(PI3K) and the serine/threonine kinase Akt, and activated mTOR phosphorylates p70 ribosomal protein S6 kinase (p70S6K) and eukaryotic initiation factor 4E-binding protein 1 (4E-BP1), both regulators of mRNA translation and cell proliferation. Rapamycin inhibits mTOR by binding to the intracellular receptor FK506-binding protein 12 . The inhibition of mTOR decreases the phosphorylation of p70S6K and 4E-BP1, and this inhibits the translation of critical mRNA sequences that are involved in cell cycle progression and cell proliferation, which are the hallmarks of carcinogenesis [3]. Clinically, rapamycin and its more stable analogues are well tolerated by patients in phase I or II trials, and have shown promising antitumor activity in several types of human cancers, including NSCLC [4-6].

However, only a minority of patients appear to respond to rapamycin analogues. Of 14 patients with advanced NSCLC treated with the rapamycin analogue, everolimus, in two phase I trials, disease stabilization was observed in only 4 , with 1 patient showing partial response $[5,6]$. Furthermore, the clinical parameters and/or molecular pathways that predict which patients will derive the greatest benefit from rapamycin analogues is unclear. These agents might have antitumor activity only in selected patients with mTOR pathway-driven disease. Antitumor therapies may therefore be improved by the use of molecular markers to select who would benefit most from treatment. In this study, we assessed the immunohistochemical expression of MTOR and its upstream and downstream molecules, including phosphorylated Akt (pAkt), p70S6K and phosphatase and tensin homologue deleted on chromosome 10 (PTEN). In addition, we investigated whether our findings are associated with clinicopathologic characteristics and patient outcome of NSCLC.

\section{Patients and Methods}

\section{Patients and Materials}

This study included 91 patients with lung adenocarcinoma (AC) and 154 patients with lung squamous cell carcinoma (SCC), who underwent surgery at our institution. The patients did not receive radiotherapy or chemotherapy before surgery. No distant metastases were identified at the time of surgery. Two independent pathologists (H.-S.K. and G.Y.K.) reviewed all hematoxylin-eosinstained slides to confirm the histologic diagnosis. Clinicopathologic data, including age, sex, histologic grade, histologic subtype of AC, tumor size, pathologic T stage ( $\mathrm{pT}$ ), pathologic $\mathrm{N}$ stage $(\mathrm{pN}), \mathrm{TNM}$ stage and postoperative follow-up, were assessed. All tumors were assessed for histologic grade according to the World Health Organization classification [1]. All cases of AC were subclassified into lepidic predominant (formerly nonmucinous bronchioloalveolar carcinoma pattern, with $>5 \mathrm{~mm}$ invasion), acinar predominant, papillary predominant, micropapillary predomi-

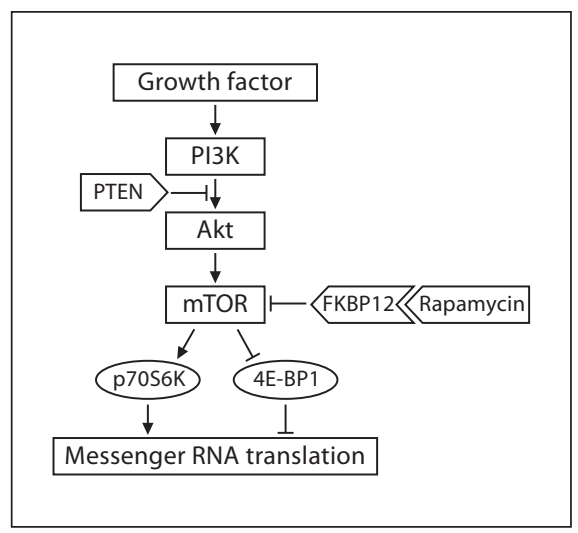

Fig. 1. PI3K-Akt-mTOR pathway. Activation of mTOR by PI3K and Akt leads to phosphorylation and activation of p70S6K and phosphorylation and inhibition of 4E-BP1. Rapamycin inhibits mTOR by binding to the FK506-binding protein 12 (FKBP12). Inhibition of mTOR decreases the phosphorylation of p70S6K and $4 \mathrm{E}-\mathrm{BP} 1$. PTEN acts as a negative regulator of this pathway.

nant, solid predominant with mucin production and invasive mucinous (formerly mucinous bronchioloalveolar carcinoma pattern), according to the International Association for the Study of Lung Cancer/American Thoracic Society/European Respiratory Society classification of lung AC in resection specimens [7]. TNM stage was postoperatively determined according to the 7 th edition of the American Joint Committee on Cancer staging system [8].

\section{Tissue Microarray Construction}

We used formalin-fixed, paraffin-embedded tissues from 91 human ACs and 154 human SCCs. The most representative tumor area without necrosis was carefully selected and marked on the hematoxylin-eosin-stained slide. The formalin-fixed, paraffin-embedded tissues corresponding to the histologic sections were sampled using a commercially available tissue microarray (TMA) instrument (AccuMax ${ }^{\mathrm{TM}}$ Arrayer, ISU ABXIS Co. Ltd., Seoul, Republic of Korea) consisting of thin-walled stainless steel punches and stylets used to empty and transfer the needle content. The assembly was held in an X-Y position guide equipped with semiautomatic micrometers, with a $1-\mathrm{mm}$ increment between individual samples and a $3-\mathrm{mm}$ punch depth stop device. The instrument was used to create holes in a recipient block with defined array cores. A solid stylet that closely fitted the needle was used to transfer the tissue cores into the recipient block. To account for the limitations of the representative tumor areas, we used triplicate 2-mm diameter tissue cores from each donor block. Multiple $4-\mu \mathrm{m}$ sections were cut with a Leica RM2255 rotary microtome (Leica Microsystems, Bannockburn, Ill., USA), and sections were transferred to adhesive-coated slides using routine histology procedures.

Immunohistochemical Staining

Immunohistochemical staining was performed using the Bond Polymer Intense Detection System (Vision BioSystems, Mount Waverley, Vic., Australia) according to the manufacturer's 
instructions. In brief, the sections were deparaffinized using Bond Dewax Solution (Vision BioSystems), and antigen retrieval was performed using Bond ER Solution (Vision BioSystems) for $30 \mathrm{~min}$ at $100^{\circ} \mathrm{C}$. Endogenous peroxidase activity was quenched by incubation with hydrogen peroxide for $5 \mathrm{~min}$. Sections were incubated for $15 \mathrm{~min}$ at ambient temperature with a rabbit polyclonal anti-pAkt (Ser473) antibody (1:250; GeneTex, Irvine, Calif., USA), a rabbit monoclonal anti-mTOR antibody (1:100; clone Y391; Epitomics, Inc., Burlingame, Calif., USA), a rabbit monoclonal anti-p70S6K antibody (1:50; clone E175; Epitomics) or a rabbit monoclonal anti-PTEN antibody (1:100; clone Y184; Epitomics). The biotin-free polymeric horseradish peroxidase-linker antibody conjugate system was used in the Bond-maX ${ }^{\mathrm{TM}}$ automatic slide stainer (Vision BioSystems) and visualization was performed by 3.3'-diaminobenzidine (DAB) solution [1 mM DAB, 50 $\mathrm{mM}$ Tris- $\mathrm{HCl}$ buffer ( $\mathrm{pH}$ 7.6) and $0.006 \% \mathrm{H}_{2} \mathrm{O}_{2}$ ]. Nuclei were counterstained with hematoxylin. Slides were subsequently dehydrated following a standard procedure and sealed with coverslips. Positive control samples were normal placental tissue for pAkt, mTOR and p70S6K, and normal lung tissue for PTEN. Negative control was prepared by substituting non-immune serum for antibody.

\section{Evaluation of Immunohistochemical Staining}

Two experienced independent pathologists (H.-S.K. and G.Y.K.), who were blinded to the clinicopathologic data and patient outcomes, examined pAkt, mTOR, p70S6K and PTEN immunostaining in TMAs and whole sections. The scores of the two pathologists were compared and discrepancies resolved through re-examination by both pathologists to achieve a consensus score. Immunoreactivity was evaluated semiquantitatively based on staining intensity and proportion, as previously described [9]. Staining intensity was scored as: absent (0); weak (1); moderate (2), or strong staining (3). Staining proportion was scored as: none (0); less than $1 / 3(1) ; 1 / 3$ to $2 / 3$ (2), or more than $2 / 3$ of tumor cells (3). The overall score was calculated as the sum of the intensity score and the proportion score, yielding a score between 0 and 6 . An overall score of $0-2$ was regarded as negative while the other scores were regarded as positive in statistical analysis. In cases of different overall scores among cores taken from the same tumor, the average score was considered the final overall score. A sample was considered positive if at least one of the three cores stained positively. A core was considered insufficient for evaluation if $<10 \%$ of the core area contained tumor tissue. If two cores were missed or were considered insufficient, the score of the remaining core was considered the final score.

\section{Statistical Analysis}

To determine whether the components of the mTOR pathway are co-expressed in tumor tissues, the relationships between each pair of proteins were assessed using the $\chi^{2}$ test, as were the relationships between each protein and clinicopathologic characteristics. Univariate and multivariate survival analyses were used to determine the prognostic significance of the expression of pAkt, mTOR, p70S6K and PTEN in patients with NSCLC. Overall survival was defined as the interval from surgery to the death of the patient. Loss to follow-up, death from a cause other than NSCLC and survival until the end of the follow-up period were regarded as censoring events. Overall survival curves were drawn according to the Kaplan-Meier method and differences were analyzed by applying the log-rank test for univariate survival analysis. Multivariate survival analysis was performed using the Cox proportional hazard models (95\% confidence interval) with a backward stepwise elimination method. All significant variables in univariate analysis were entered into the multivariate analysis. The least significant variables were removed from the model by backward stepwise elimination. Statistical analyses were performed using SPSS version 15.0 (SPSS Inc., Chicago, Ill., USA). Statistical significance was defined as $\mathrm{p}<0.05$.

\section{Results}

\section{Demographic and Clinicopathologic Characteristics}

Of 245 cases of NSCLC, 194 (79.2\%) were men, and 51 were $(20.8 \%)$ women. The median age of patients was 64 years (range, $35-81$ ), and $46.9 \%$ (115/245) were aged $\geq 64$ years. Nodal metastases were detected in 106 (43.3\%) patients. The TNM stage was I in 89 (36.3\%) patients, II in $78(31.8 \%)$ and III in 78 (31.8\%). Selected operative procedures were lobectomy in 201 patients, pneumonectomy in 23 patients, bilobectomy in 19 patients and sleeve lobectomy in 2 patients. Follow-up information was available for all 245 NSCLC patients for periods ranging from 1 to 280 months (median, 39 months). A total of 32 of 91 (35.2\%) AC patients and 83 of 154 (53.9\%) SCC patients had died at the time of the last follow-up. Other baseline clinicopathologic characteristics of $91 \mathrm{AC}$ patients and 154 SCC patients are given in table 1 .

\section{Validation of the TMA Method and Comparison of \\ TMA and Whole-Section Immunostaining}

No core was considered insufficient for evaluation. No case was excluded from analysis, although out of 245 cases of NSCLC, one of three cores was lost in 7 (2.9\%) cases, and two of three were lost in $3(1.2 \%)$ cases during block processing, sectioning and immunostaining. Immunohistochemistry for four proteins was performed on whole sections of randomly selected original tumor tissue blocks (10 ACs and 10 SCCs) to compare the results of immunostained TMAs with those of whole sections. The expression of all four proteins was homogeneous throughout the tumor, and showed complete concordance between TMAs and whole sections in all selected cases.

\section{Immunohistochemical Expression of pAkt, mTOR, p70S6K and PTEN and Their Correlations}

Representative immunostaining for each protein in AC and SCC is illustrated in figures 2 and 3. pAkt expression was observed in 200 (81.6\%) patients in both the cytoplasm and the nucleus (fig. 2a, 3a). mTOR was detected 
Table 1. Baseline clinicopathologic characteristics of 91 patients with AC and 154 patients with SCC

\begin{tabular}{|c|c|c|}
\hline \multirow[t]{2}{*}{ Characteristics } & \multicolumn{2}{|c|}{ Cases, n (\%) } \\
\hline & $\mathrm{AC}$ & SCC \\
\hline \multicolumn{3}{|l|}{ Age } \\
\hline$\geq 64$ years & $41(45.1)$ & $74(48.1)$ \\
\hline$<64$ years & $50(54.9)$ & $80(51.9)$ \\
\hline \multicolumn{3}{|l|}{ Sex } \\
\hline Male & $55(60.4)$ & $139(90.3)$ \\
\hline Female & $36(39.6)$ & $15(9.7)$ \\
\hline \multicolumn{3}{|l|}{ Histologic grade } \\
\hline 1 (well differentiated) & $9(20.9)$ & $8(5.2)$ \\
\hline 2 (moderately differentiated) & $63(69.2)$ & $126(81.8)$ \\
\hline 3 (poorly differentiated) & $19(20.9)$ & $20(13.0)$ \\
\hline \multicolumn{3}{|l|}{ Histologic subtype } \\
\hline Lepidic predominant & $17(6.9)$ & not applicable \\
\hline Acinar predominant & $65(26.5)$ & \\
\hline Papillary predominant & $4(1.6)$ & \\
\hline Micropapillary predominant & 0 & \\
\hline Solid predominant with & & \\
\hline mucin production & $3(1.2)$ & \\
\hline Invasive mucinous & $2(0.8)$ & \\
\hline \multicolumn{3}{|l|}{ Tumor size } \\
\hline$<4.5 \mathrm{~cm}$ & $27(29.7)$ & $70(45.5)$ \\
\hline$\geq 4.5 \mathrm{~cm}$ & $64(70.3)$ & $84(54.5)$ \\
\hline \multicolumn{3}{|l|}{ Pathologic tumor stage } \\
\hline $\mathrm{pT}_{1}$ & $25(27.5)$ & $24(15.6)$ \\
\hline $\mathrm{pT}_{2}$ & $47(51.6)$ & $83(53.9)$ \\
\hline $\mathrm{pT}_{3}$ & $15(16.5)$ & $35(22.7)$ \\
\hline $\mathrm{pT}_{4}$ & $4(4.4)$ & $12(7.8)$ \\
\hline \multicolumn{3}{|l|}{ Pathologic node stage } \\
\hline $\mathrm{pN}_{0}$ & $28(30.8)$ & $92(59.7)$ \\
\hline $\mathrm{pN}_{1}$ & $16(17.6)$ & $38(24.7)$ \\
\hline $\mathrm{pN}_{2}$ & $8(30.8)$ & $24(15.6)$ \\
\hline $\mathrm{pN}_{3}$ & 0 & 0 \\
\hline \multicolumn{3}{|l|}{ TNM stage } \\
\hline IA & $18(19.8)$ & $17(11.0)$ \\
\hline IB & $18(19.8)$ & $36(23.4)$ \\
\hline IIA & $10(11.0)$ & $26(16.9)$ \\
\hline IIB & $11(12.1)$ & $31(20.1)$ \\
\hline IIIA & $31(34.1)$ & $42(27.3)$ \\
\hline IIIB & $3(3.3)$ & $2(1.3)$ \\
\hline
\end{tabular}

Histologic subtypes were according to IASLC/ATS/ERS. Classification of lung AC in resection specimens [7].

in $161(65.7 \%)$ patients and was exclusively cytoplasmic (fig. 2b, 3b). p70S6K was also expressed in both the cytoplasm and the nucleus in 179 (73.1\%) patients (fig. 2c, 3c). Normal lung tissue showed no immunoreactivity to pAkt, mTOR or p70S6K. PTEN was expressed in normal pneumocytes and located in the cytoplasmic cellular component. In contrast, in tumor tissues, PTEN expression was lost; 135 (55.1\%) cases were positive for PTEN (fig. 2d) and 110 (44.9\%) cases were negative for PTEN (fig. 3d). As shown in table 2, in NSCLC, pAkt expression was positively associated with the expression of mTOR $(\mathrm{p}<0.001)$ and $\mathrm{p} 70 \mathrm{S6} \mathrm{K}(\mathrm{p}<0.001)$. mTOR expression was also positively associated with p70S6K expression ( $\mathrm{p}<$ $0.001)$. PTEN expression was inversely correlated with the expression of pAkt $(\mathrm{p}=0.001)$, mTOR $(\mathrm{p}<0.001)$ and p70S6K $(\mathrm{p}=0.012)$. Likewise, in both AC and SCC, the relationships between each pair of proteins were also statistically significant.

Associations of the Expression of pAkt, mTOR, p70S6K and PTEN with Clinicopathologic Characteristics

For AC (table 3), loss of PTEN expression, observed in $37.4 \%$ (34/91) of cases, was significantly associated with aggressive tumor features, including a higher histologic grade $(\mathrm{p}=0.013)$, $\mathrm{pT}(\mathrm{p}=0.016)$ and $\mathrm{pN}(\mathrm{p}<0.001)$, as well as an advanced TNM stage $(\mathrm{p}=0.001)$. In contrast, we found no association between the expression of pAkt, mTOR or p70S6K and clinicopathologic characteristics of AC. For SCC (table 4), none of the clinicopathologic characteristics showed a significant relationship with the expression of pAkt, mTOR, p70S6K or PTEN.

\section{Prognostic Significance of the Expression of pAkt, mTOR, p70S6K and PTEN}

For AC (table 5), univariate analysis revealed that an advanced TNM stage $(\mathrm{p}=0.014)$ and loss of PTEN expression $(\mathrm{p}=0.015)$ were significant predictors for $\mathrm{a}$ shorter overall survival. The median survival of AC patients with negative PTEN expression was 45 months. The median survival of the patients whose AC was positive for PTEN could not be calculated because the survival curve never decreased below 50\%. The 1-, 3-, 5and 10 -year survival rates were $84.5,67.9,49.5$ and $33.4 \%$ for patients with PTEN-negative AC and 100.0, 80.1, 75.0 and $60.4 \%$ for patients with PTEN-positive AC (fig. 4a). On multivariate analysis, neither TNM stage nor PTEN expression independently predicted patient outcome.

For SCC (table 5), univariate analysis showed that an older age $(\mathrm{p}=0.017)$, a higher $\mathrm{pT}(\mathrm{p}<0.001)$ and $\mathrm{pN}(\mathrm{p}=$ $0.001)$ and an advanced TNM stage $(\mathrm{p}<0.001)$ were significant predictors of shorter overall survival. Negative PTEN expression in SCC correlated with a shortened median survival of 64 months, compared with $>10$ years (121 months) in patients with PTEN-positive SCC, but the difference between the survival curves was not sig- 
Fig. 2. Representative positive immunoreactivity for pAkt (a), mTOR (b), p70S6K (c) and PTEN (d) in lung AC. Polymer method; original magnification, $\times 200$.
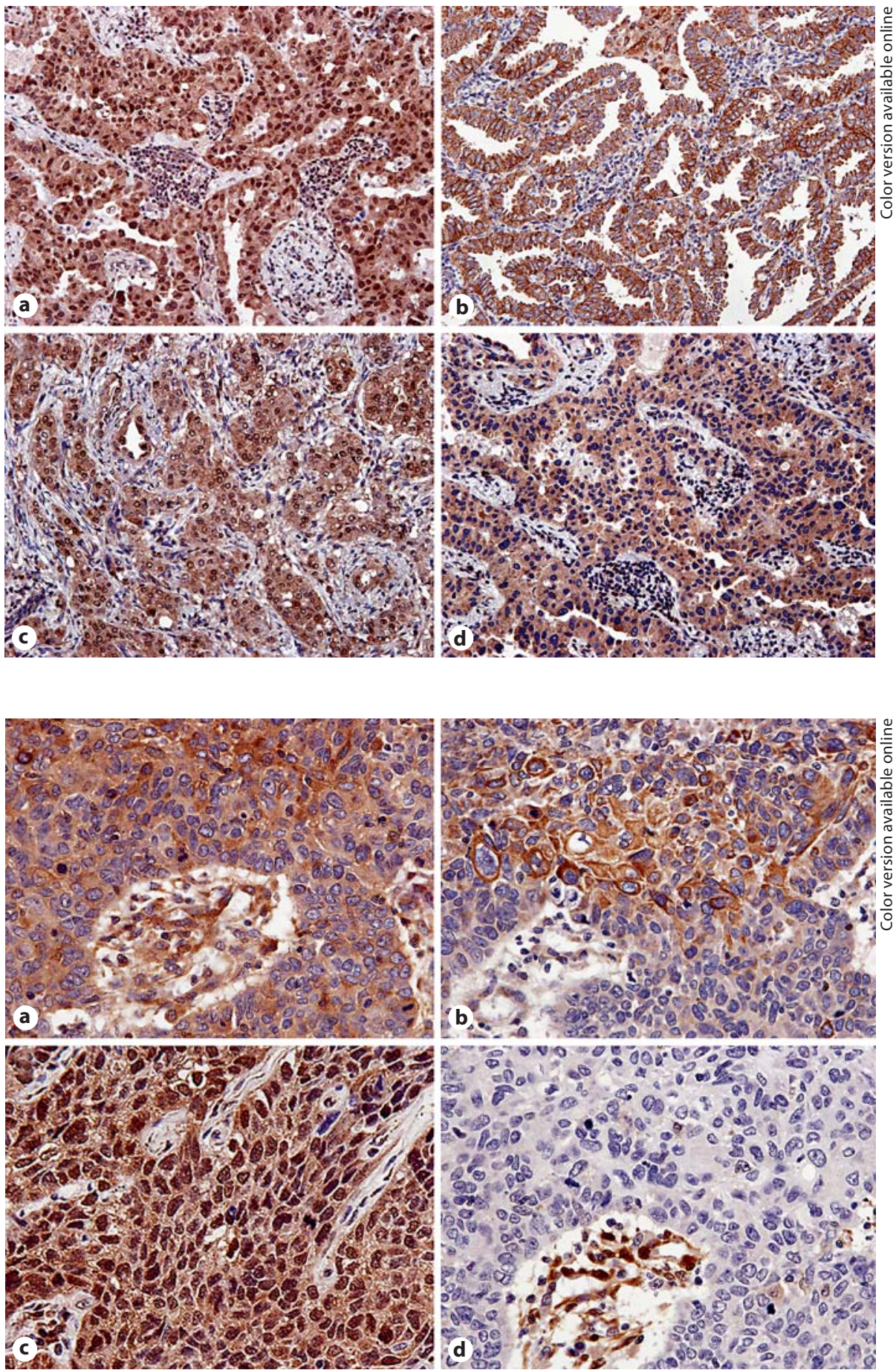

Fig. 3. Representative positive immunoreactivity for pAkt (a), mTOR (b), p70S6K (c) and negative immunoreactivity for PTEN (d) in lung SCC. Polymer method; original magnification, $\times 400$.

\section{Discussion}

The tumor suppressor PTEN is located on chromosome 10q23 and functions as a lipid phosphatase that dephosphorylates phosphatidylinositol $(3,4,5)$-triphos- 
Table 2. Correlations between the expression of pAkt, mTOR, p70S6K and PTEN in 245 cases with NSCLC

\begin{tabular}{|c|c|c|c|c|c|c|c|c|c|c|c|c|}
\hline \multirow[t]{2}{*}{ Markers } & \multicolumn{3}{|l|}{ pAkt } & \multicolumn{3}{|l|}{ mTOR } & \multicolumn{3}{|l|}{ p70S6K } & \multicolumn{3}{|l|}{ PTEN } \\
\hline & $+(\%)$ & $-(\%)$ & $\mathrm{p}$ value & $+(\%)$ & $-(\%)$ & $\mathrm{p}$ value & $+(\%)$ & $-(\%)$ & $p$ value & $+(\%)$ & $-(\%)$ & $\mathrm{p}$ value \\
\hline \multicolumn{13}{|l|}{ pAkt } \\
\hline+ & & NA & & $144(89.4)$ & $56(28.0)$ & $<0.001$ & $136(84.5)$ & 25 (15.5) & $<0.001$ & $72(44.7)$ & $89(55.3)$ & $<0.001$ \\
\hline _- & & & & $17(37.8)$ & $28(33.3)$ & & $43(51.2)$ & $41(48.8)$ & & $63(75.0)$ & $21(25.0)$ & \\
\hline \multicolumn{13}{|l|}{ TOR } \\
\hline+ & $144(72.0)$ & $17(10.6)$ & $<0.001$ & & NA & & $164(82.0)$ & $36(18.0)$ & $<0.001$ & $100(50.0)$ & $100(50.0)$ & 0.001 \\
\hline - & $56(66.7)$ & $28(33.3)$ & & & & & $15(33.3)$ & $30(66.7)$ & & $35(77.8)$ & $10(22.2)$ & \\
\hline \multicolumn{13}{|l|}{ p70S6K } \\
\hline+ & 164 (91.6) & $15(8.4)$ & $<0.001$ & $136(76.0)$ & $43(24.0)$ & $<0.001$ & & NA & & $90(50.3)$ & 89 (49.7) & 0.012 \\
\hline- & $36(54.5)$ & $30(45.5)$ & & $25(37.9)$ & $41(62.1)$ & & & & & $45(31.8)$ & $21(68.2)$ & \\
\hline \multicolumn{13}{|l|}{ PTEN } \\
\hline+ & $100(74.1)$ & 35 (25.9) & 0.001 & $72(53.3)$ & $63(46.7)$ & $<0.001$ & $90(66.7)$ & $45(33.3)$ & 0.012 & & NA & \\
\hline - & $100(90.9)$ & $10(9.1)$ & & $89(80.9)$ & $21(19.1)$ & & $89(80.9)$ & $21(19.1)$ & & & & \\
\hline
\end{tabular}

NA = Not applicable.

Table 3. Associations of pAkt, mTOR, p70S6K and PTEN expression with clinicopathologic characteristics of 91 patients with AC

\begin{tabular}{|c|c|c|c|c|c|c|c|c|c|c|c|c|}
\hline \multirow[t]{2}{*}{ Characteristics } & \multicolumn{3}{|l|}{ pAkt } & \multicolumn{3}{|l|}{ mTOR } & \multicolumn{3}{|l|}{ p70S6K } & \multicolumn{3}{|l|}{ PTEN } \\
\hline & $+(\%)$ & $-(\%)$ & $\mathrm{p}$ value & $+(\%)$ & $-(\%)$ & $\mathrm{p}$ value & $+(\%)$ & $-(\%)$ & $\mathrm{p}$ value & $+(\%)$ & $-(\%)$ & $\mathrm{p}$ value \\
\hline \multicolumn{13}{|l|}{ Age } \\
\hline$\geq 64$ years & $35(85.4)$ & $6(14.6)$ & \multirow[t]{2}{*}{0.931} & $27(65.9)$ & $14(34.1)$ & \multirow[t]{2}{*}{0.182} & $33(76.7)$ & $8(19.5)$ & \multirow[t]{2}{*}{0.252} & $28(68.3)$ & $13(31.7)$ & \multirow[t]{2}{*}{0.313} \\
\hline$<64$ years & $43(86.0)$ & $7(14.0)$ & & $26(52.0)$ & $24(48.0)$ & & $35(70.0)$ & $15(30.0)$ & & $29(58.0)$ & $21(42.0)$ & \\
\hline \multicolumn{13}{|l|}{ Sex } \\
\hline Male & $45(81.8)$ & $10(18.2)$ & \multirow[t]{2}{*}{0.233} & $31(56.4)$ & $24(43.6)$ & \multirow[t]{2}{*}{0.653} & $42(76.4)$ & $13(23.6)$ & \multirow[t]{2}{*}{0.657} & $36(65.5)$ & $19(34.5)$ & \multirow[t]{2}{*}{0.492} \\
\hline Female & 33 (91.7) & $3(8.3)$ & & $22(61.1)$ & $14(38.9)$ & & $26(72.2)$ & $10(27.8)$ & & $21(58.3)$ & $15(41.7)$ & \\
\hline \multicolumn{13}{|l|}{ Histologic grade } \\
\hline Well & $9(100.0)$ & 0 & \multirow{3}{*}{0.754} & $4(44.4)$ & $5(55.6)$ & \multirow[t]{3}{*}{0.398} & $8(88.9)$ & $1(11.1)$ & \multirow[t]{3}{*}{0.516} & $8(88.9)$ & $1(11.1)$ & \multirow[t]{3}{*}{0.013} \\
\hline Moderate & $52(82.5)$ & $11(17.5)$ & & $37(58.7)$ & $26(41.3)$ & & $46(73.0)$ & $17(27.0)$ & & $41(65.1)$ & $22(34.9)$ & \\
\hline Poor & $17(89.5)$ & $2(10.5)$ & & $12(63.2)$ & 7 (36.8) & & $14(73.7)$ & $5(26.3)$ & & $8(42.1)$ & $11(57.9)$ & \\
\hline \multicolumn{13}{|c|}{ Histologic subtype } \\
\hline LP & $14(82.4)$ & $3(17.6)$ & \multirow{6}{*}{0.892} & $11(64.7)$ & $6(35.3)$ & \multirow[t]{6}{*}{0.941} & $12(70.6)$ & $5(29.4)$ & \multirow[t]{6}{*}{0.441} & $12(70.6)$ & $5(29.4)$ & \multirow[t]{6}{*}{0.729} \\
\hline $\mathrm{AP}$ & $57(87.7)$ & $8(12.3)$ & & $36(55.4)$ & $29(44.6)$ & & $48(73.8)$ & $17(26.2)$ & & $40(61.5)$ & $25(38.5)$ & \\
\hline $\mathrm{PP}$ & $3(75.0)$ & $1(25.0)$ & & $3(75.0)$ & $1(25.0)$ & & $4(100.0)$ & 0 & & $2(50.0)$ & $2(50.0)$ & \\
\hline MP & 0 & 0 & & 0 & 0 & & 0 & 0 & & 0 & 0 & \\
\hline SP & $2(66.7)$ & $1(33.3)$ & & $2(66.7)$ & $1(33.3)$ & & $2(66.7)$ & $1(33.3)$ & & $1(33.3)$ & $2(66.7)$ & \\
\hline $\mathrm{IM}$ & $2(100.0)$ & 0 & & $1(50.0)$ & $1(50.0)$ & & $2(100.0)$ & 0 & & $2(100.0)$ & 0 & \\
\hline Tumor size & & & & & & & & & & & & \\
\hline$<4.5 \mathrm{~cm}$ & $22(81.5)$ & $5(18.5)$ & 0.454 & $13(48.1)$ & $14(51.9)$ & 0.205 & $17(63.0)$ & $1037.0)$ & 0.094 & $16(59.3)$ & $11(40.7)$ & 0.665 \\
\hline$\geq 4.5 \mathrm{~cm}$ & $56(87.5)$ & $8(12.5)$ & & $40(62.5)$ & $24(37.5)$ & & $51(79.7)$ & $1320.3)$ & & $41(64.1)$ & 23 (35.9) & \\
\hline pT & & & & & & & & & & & & \\
\hline $\mathrm{pT}_{1}$ & $23(92.0)$ & $2(8.0)$ & 0.212 & $15(60.0)$ & $10(40.0)$ & 0.301 & $21(84.0)$ & $4(16.0)$ & 0.168 & $20(80.0)$ & $5(20.0)$ & 0.016 \\
\hline $\mathrm{pT}_{2}$ & $40(85.1)$ & $7(14.9)$ & & $30(63.8)$ & $17(36.2)$ & & $34(72.3)$ & $13(27.7)$ & & $27(57.4)$ & $20(42.6)$ & \\
\hline $\mathrm{pT}_{3}$ & $12(80.0)$ & $3(20.0)$ & & $6(40.0)$ & $9(60.0)$ & & $11(73.3)$ & $4(26.7)$ & & $10(66.7)$ & $5(33.3)$ & \\
\hline $\mathrm{pT}_{4}$ & $3(75.0)$ & $1(25.0)$ & & $2(50.0)$ & $2(50.0)$ & & $2(50.0)$ & $2(50.0)$ & & 0 & $4(100.0)$ & \\
\hline $\mathrm{pN}$ & & & & & & & & & & & & \\
\hline $\mathrm{pN}_{0}$ & $40(92.9)$ & $7(14.9)$ & 0.441 & $27(57.4)$ & $20(42.6)$ & 0.331 & 38 (80.9) & $9(19.1)$ & 0.447 & $36(76.6)$ & $11(23.4)$ & 0.001 \\
\hline $\mathrm{pN}_{1}$ & $12(75.0)$ & $4(25.0)$ & & $6(37.5)$ & $10(62.5)$ & & $9(56.3)$ & $7(43.8)$ & & $12(75.0)$ & $4(25.0)$ & \\
\hline $\mathrm{pN}_{2}$ & $26(92.9)$ & $2(7.1)$ & & $20(71.4)$ & $8(28.6)$ & & $21(75.0)$ & $7(25.0)$ & & $9(32.1)$ & $19(67.9)$ & \\
\hline TNM stage & & & & & & & & & & & & \\
\hline I & $32(88.9)$ & $4(11.1)$ & 0.808 & $23(63.9)$ & $13(36.1)$ & 0.840 & $29(80.6)$ & 7 (19.4) & 0.338 & $27(75.0)$ & $9(25.0)$ & 0.001 \\
\hline II & $15(71.4)$ & $6(28.6)$ & & $9(42.9)$ & $12(57.1)$ & & $15(71.4)$ & $6(28.6)$ & & $18(85.7)$ & $3(14.3)$ & \\
\hline III & 31 (91.2) & $3(8.8)$ & & $21(61.8)$ & $13(38.2)$ & & $24(70.6)$ & $10(29.4)$ & & $12(35.3)$ & $22(64.7)$ & \\
\hline
\end{tabular}

$\mathrm{LP}=$ Lepidic predominant; $\mathrm{AP}=$ acinar predominant; $\mathrm{PP}=$ papillary predominant; $\mathrm{MPP}=$ micropapillary predominant; $\mathrm{SP}=$ solid predominant with mucin production; $\mathrm{IM}=$ invasive mucinous. 
Table 4. Associations of pAkt, mTOR, p70S6K and PTEN expression with clinicopathologic characteristics of 154 patients with SCC

\begin{tabular}{|c|c|c|c|c|c|c|c|c|c|c|c|c|}
\hline \multirow[t]{2}{*}{ Characteristics } & \multicolumn{3}{|l|}{ pAkt } & \multicolumn{3}{|l|}{ mTOR } & \multicolumn{3}{|l|}{ p70S6K } & \multicolumn{3}{|l|}{ PTEN } \\
\hline & $+(\%)$ & $-(\%)$ & $\mathrm{p}$ value & $+(\%)$ & $-(\%)$ & $\mathrm{p}$ value & $+(\%)$ & $-(\%)$ & $\mathrm{p}$ value & $+(\%)$ & $-(\%)$ & $\mathrm{p}$ value \\
\hline \multicolumn{13}{|l|}{ Age } \\
\hline$\geq 64$ years & $57(77.0)$ & $17(23.0)$ & 0.519 & $48(64.9)$ & $26(35.1)$ & 0.170 & $51(68.9)$ & $23(31.1)$ & 0.401 & $42(56.8)$ & $32(43.2)$ & 0.145 \\
\hline$<64$ years & $65(81.3)$ & $15(18.8)$ & & $60(75.0)$ & $20(25.0)$ & & $60(75.0)$ & $20(25.0)$ & & $36(45.0)$ & $44(55.0)$ & \\
\hline \multicolumn{13}{|l|}{ Sex } \\
\hline Male & $108(77.7)$ & $31(22.3)$ & 0.198 & $97(69.8)$ & $42(30.2)$ & 0.775 & $100(71.9)$ & $39(28.1)$ & 1.000 & $68(48.9)$ & $71(51.1)$ & 0.192 \\
\hline Female & $14(93.3)$ & $1(6.7)$ & & $11(73.3)$ & $4(26.7)$ & & $11(73.3)$ & $4(26.7)$ & & $10(66.7)$ & $5(33.3)$ & \\
\hline \multicolumn{13}{|l|}{ Histologic grade } \\
\hline Well & $7(87.5)$ & $1(12.5)$ & 0.816 & $6(75.0)$ & $2(25.0)$ & 0.312 & $8(100.0)$ & 0 & 0.481 & $5(62.5)$ & $3(37.5)$ & 0.680 \\
\hline Moderate & $98(77.8)$ & $28(22.2)$ & & $90(71.4)$ & $36(28.6)$ & & $88(69.8)$ & $38(30.2)$ & & $63(50.0)$ & $63(50.0)$ & \\
\hline Poor & $17(85.0)$ & $3(15.0)$ & & $12(60.0)$ & $8(40.0)$ & & $15(75.0)$ & $5(25.0)$ & & $10(50.0)$ & $10(50.0)$ & \\
\hline \multicolumn{13}{|l|}{ Tumor size } \\
\hline$<4.5 \mathrm{~cm}$ & $53(75.7)$ & $17(24.3)$ & 0.328 & $46(65.7)$ & $24(34.3)$ & 0.274 & $48(68.6)$ & $22(31.4)$ & 0.376 & $38(54.3)$ & $32(45.7)$ & 0.410 \\
\hline$\geq 4.5 \mathrm{~cm}$ & $69(82.1)$ & 15 (17.9) & & $62(73.8)$ & $22(26.2)$ & & $63(75.0)$ & $21(25.0)$ & & $40(47.6)$ & $44(52.4)$ & \\
\hline \multicolumn{13}{|l|}{$\mathrm{pT}$} \\
\hline $\mathrm{pT}_{1}$ & $24(100.0)$ & 0 & 0.946 & $18(75.0)$ & $6(25.0)$ & 0.330 & $20(83.3)$ & 4(16.7) & 0.471 & $13(54.2)$ & $11(45.8)$ & 0.178 \\
\hline $\mathrm{pT}_{2}$ & $58(69.9)$ & $25(30.1)$ & & $54(65.1)$ & $29(34.9)$ & & $57(68.7)$ & $26(31.3)$ & & $42(50.6)$ & $41(49.4)$ & \\
\hline $\mathrm{pT}_{3}$ & $28(80.0)$ & $7(20.0)$ & & $25(71.4)$ & $10(28.6)$ & & $26(74.3)$ & $9(25.7)$ & & $22(62.9)$ & $13(37.1)$ & \\
\hline $\mathrm{pT}_{4}$ & $12(100.0)$ & 0 & & $11(91.7)$ & $1(8.3)$ & & $8(66.7)$ & $4(33.3)$ & & $1(8.3)$ & $11(91.7)$ & \\
\hline \multicolumn{13}{|l|}{$\mathrm{pN}^{\mathrm{r}}$} \\
\hline $\mathrm{pN}_{0}$ & $74(80.4)$ & $18(19.6)$ & 0.573 & $66(71.7)$ & $26(28.3)$ & 0.437 & $63(68.5)$ & $29(31.5)$ & 0.336 & 45 (48.9) & $47(51.1)$ & 0.904 \\
\hline $\mathrm{pN}_{1}$ & $30(78.9)$ & $8(21.1)$ & & $27(71.1)$ & $11(28.9)$ & & $30(78.9)$ & $8(21.1)$ & & $23(60.5)$ & $15(39.5)$ & \\
\hline $\mathrm{pN}_{2}$ & $18(75.0)$ & $6(25.0)$ & & $15(62.5)$ & $9(37.5)$ & & $18(75.0)$ & $6(25.0)$ & & $10(41.7)$ & $14(58.3)$ & \\
\hline \multicolumn{13}{|l|}{ TNM stage } \\
\hline I & $43(81.1)$ & $10(18.9)$ & 0.974 & $39(73.6)$ & $14(26.4)$ & 0.945 & $40(75.5)$ & $13(24.5)$ & 0.908 & $26(49.1)$ & $27(50.9)$ & 0.367 \\
\hline II & $43(75.4)$ & $14(24.6)$ & & $36(63.2)$ & $21(36.8)$ & & $38(66.7)$ & $19(33.3)$ & & $35(61.4)$ & $22(38.6)$ & \\
\hline III & $36(81.8)$ & $8(18.2)$ & & $33(75.0)$ & $11(25.0)$ & & $33(75.0)$ & $11(25.0)$ & & $17(38.6)$ & $27(61.4)$ & \\
\hline
\end{tabular}

Table 5. Factors predicting worse outcome of 91 patients with AC and 154 patients with SCC (univariate and multivariate survival analyses)

\begin{tabular}{|c|c|c|c|c|c|c|}
\hline \multirow[t]{3}{*}{ Characteristics } & \multicolumn{3}{|l|}{$\mathrm{AC}$} & \multicolumn{3}{|l|}{ SCC } \\
\hline & \multirow{2}{*}{$\frac{\text { univariate }}{\text { p value }}$} & \multicolumn{2}{|l|}{ multivariate } & \multirow{2}{*}{$\frac{\text { univariate }}{\mathrm{p} \text { value }}$} & \multicolumn{2}{|l|}{ multivariate } \\
\hline & & HR (95\% CI) & $\mathrm{p}$ value & & $\operatorname{HR}(95 \% \mathrm{CI})$ & $\mathrm{p}$ value \\
\hline Age, $\geq 64$ vs. $<64$ years & 0.750 & NA & & 0.017 & $1.677(1.085-2.593)$ & 0.020 \\
\hline Sex, males vs. females & 0.773 & NA & & 0.065 & NA & \\
\hline Histologic grade, moderate-poor vs. well & 0.557 & NA & & 0.477 & NA & \\
\hline Histologic subtype, LP vs. non-LP & 0.693 & NA & & NA & & \\
\hline Tumor size, $\geq 4.5$ vs. $<4.5 \mathrm{~cm}$ & 0.845 & NA & & 0.314 & NA & \\
\hline Pathologic $\mathrm{T}$ stage, $\mathrm{pT}_{3-4}$ vs. $\mathrm{pT}_{1-2}$ & 0.116 & NA & & $<0.001$ & $2.355(1.494-3.715)$ & $<0.001$ \\
\hline Pathologic $\mathrm{N}$ stage, $\mathrm{pN}_{1-2}$ vs. $\mathrm{pN}_{0}$ & 0.203 & NA & & $<0.001$ & $2.192(1.411-3.403)$ & $<0.001$ \\
\hline TNM stage group, III vs. I-II & 0.014 & $1.917(0.904-4.065)$ & 0.090 & $<0.001$ & $1.499(0.796-2.824)$ & 0.210 \\
\hline pAkt expression, positive vs. negative & 0.591 & NA & & 0.519 & NA & \\
\hline mTOR expression, positive vs. negative & 0.528 & NA & & 0.749 & NA & \\
\hline p706SK expression, positive vs. negative & 0.451 & NA & & 0.767 & NA & \\
\hline PTEN expression, negative vs. positive & 0.015 & $1.914(0.912-4.016)$ & 0.086 & 0.141 & NA & \\
\hline
\end{tabular}

$\mathrm{HR}=$ Hazard ratio; $\mathrm{CI}=$ confidence interval; $\mathrm{NA}=$ not applicable; $\mathrm{LP}=$ lepidic predominant. 
Fig. 4. Kaplan-Meier curves for the relationship between overall survival and PTEN expression in AC (a) and SCC (b). $\mathrm{p}=0.015, \mathrm{p}=0.141$.

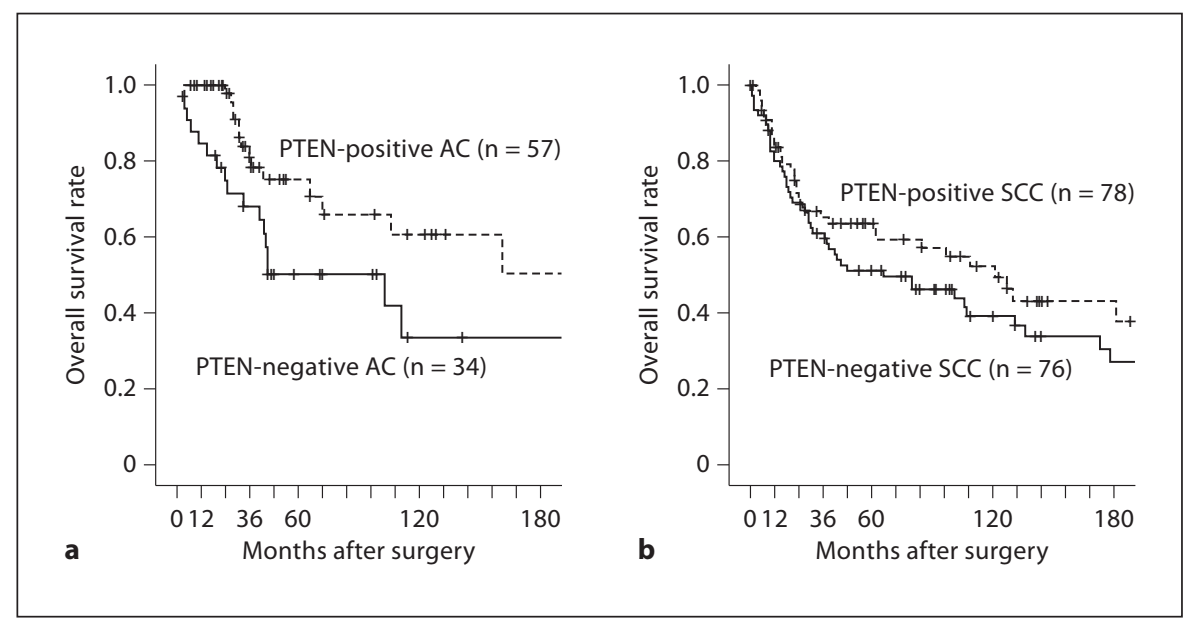

phates, a product of $\mathrm{PI} 3 \mathrm{~K}$, thereby negatively regulating the PI3K/Akt/mTOR pathway [10]. The PTEN gene is thought to function in apoptosis, cell cycle arrest and cell migration, and is frequently mutated and homozygously deleted in human neoplasms $[11,12]$. Loss of PTEN results in increased Akt activity and continued cell survival and cell proliferation. In this study, we observed loss of PTEN expression in $37.4 \%$ of ACs. In addition, we found a significant association between loss of PTEN expression and higher histologic grade, $\mathrm{pT}$ and $\mathrm{pN}$ and an advanced TNM stage, as well as shorter overall survival. These results were consistent with those of Tang et al. [13] and Yoo et al. [14] who demonstrated an association between loss of PTEN expression and the presence of nodal metastasis, an advanced TNM stage and poor patient outcome in NSCLC. Furthermore, in addition to NSCLC [13, 15], reduction and loss of PTEN expression has been reported in primary malignancies including glioblastomas, endometrial carcinomas and breast carcinomas [16-18]. The high prevalence of PTEN loss and its association with aggressive tumor behavior in AC suggests that PTEN is an underlying molecular abnormality involved in $\mathrm{AC}$ progression and that PTEN can be a promising therapeutic target for AC. In addition, a significant association between loss of PTEN expression and poor patient outcome in patients with AC suggests that PTEN expression could serve as a prognostic marker for AC.

We demonstrated that pAkt expression was positively correlated with the expression of mTOR and p70S6K, and mTOR expression was positively correlated with p70S6K expression. These results are in accordance with pAkt activating mTOR, and mTOR and pAkt being positive regulators of p70S6K $[9,19]$. We further observed that PTEN expression was inversely correlated with the expression of pAkt, mTOR and p70S6K. Previous studies suggested that the status of the mTOR pathway components is an important determinant for tumor response to mTOR inhibitors. In a preclinical breast cancer model, cancer cells that lost PTEN were found to be more sensitive to the rapamycin analogue CCI-779, and rapamycin-sensitive cell lines contained higher levels of the activated form of Akt and p70S6K [20]. A study using mouse and human cancer cell lines showed that PTEN-deficient cells are sensitive to growth inhibition caused by pharmacologic mTOR blockade by CCI-779, and that loss of PTEN is associated with increased p70S6K activity [21]. A recent study also demonstrated that overexpression of p70S6K and phosphorylation of Akt are associated with rapamycin sensitivity, supporting the proposal that p70S6K overexpression and pAkt status should be evaluated as predictors of rapamycin sensitivity [22]. These studies suggested that since an increase in the level and phosphorylation state of Akt and p70S6K is accompanied by the loss of PTEN, pAkt and p70S6K provide other determinants to predict the tumor response to mTOR inhibition. Additionally, Noh et al. [9] found that high pAkt expression, high p70S6K expression and low PTEN expression render tumors sensitive to mTOR inhibition. In this study, when positive pAkt expression, positive $\mathrm{p} 70 \mathrm{~S} 6 \mathrm{~K}$ expression and negative PTEN expression were applied as the criteria to select candidates for rapamycin treatment, 80 of 245 (32.7\%) NSCLCs, including 22 of 91 (24.2\%) ACs and 58 of 154 (37.7\%) SCCs, were found to be potential candidates. Further studies of this issue in larger cohorts are needed to provide a reliable estimate of the proportion of rapamycin-sensitive NSCLCs. 
The serine/threonine kinase Akt plays an essential role in various cellular processes, including cell growth and proliferation, metabolism and cell survival. Immunohistochemical analyses have shown that Akt is activated in many types of carcinoma and preneoplastic lesions, and Akt activation is associated with tumor progression and poor prognosis in various human malignancies [23-27]. However, in NSCLCs, the results so far are conflicting. Dobashi et al. [28] demonstrated that pAkt expression was associated with the presence of nodal metastasis in NSCLCs. Hiramatsu et al. [29] also showed that pAkt expression was associated with the presence of nodal metastasis, a higher stage and poor prognosis in lung ACs. In contrast, in a study by Balsara et al. [30], pAkt expression did not correlate with histologic grade, stage or patient survival in NSCLCs. Consistent with this result, we observed that pAkt expression was not associated with histologic grade, histologic subtype, tumor size, pT, pN or stage in ACs or SCCs. Furthermore, the positive rates for pAkt were inconsistent. In the present study, pAkt expression was positive in 85.7 and $79.2 \%$ of AC and SCC cases, respectively. In previous studies, the positive rate for pAkt were 36.2 and $49.1 \%$ of $\mathrm{AC}$ and SCC cases, respectively [28]. Concerning NSCLC cases, the positive rates ranged from 50.9 to $81.6 \%[29,30]$. The following reasons might underlie such a discrepancy: differences in sample size, intrinsic tumor heterogeneity, antibodies used, use of antigen retrieval and staining procedures with varying degrees of sensitivity and lack of a standard evaluation method for immunohistochemical staining. Our results should be confirmed in a larger cohort of patients using a constant evaluation method.

Despite results in model systems, the clinical antitumor activity of rapamycin analogues in patients has been modest [31], and only a fraction of patients appear to respond [32]. The treatment of multiple NSCLC cell lines with everolimus and rapamycin leads to down-regulation of p70S6K, but paradoxically can lead to up-regulation of Akt phosphorylation $[33,34]$. This has been attributed to the unanticipated ability of rapamycin to increase Akt activity via release of feedback inhibition. An increase in pAkt promotes tumor survival and clearly limits the potential antitumor activity of single-agent treatment with rapamycin. Preclinical evidence suggests that additive or synergistic interactions in NSCLC may be obtained with a combination of epidermal growth factor receptor-tyrosine kinase inhibitor (EGFR-TKI) and mTOR inhibitor. Buck et al. [33] observed that a combination of the EGFRTKI erlotinib and rapamycin down-regulates rapamycinstimulated pAkt activity. Similarly, O’Reilly et al. [35] demonstrated that insulin-like growth factor-I receptor inhibition prevents rapamycin-induced Akt activation and sensitizes tumor cells to inhibition by mTOR, suggesting that combination therapy can abrogate Akt induction and enhance the antitumor effects of rapamycin. Further clinical evaluations are underway for everolimus in combination with erlotinib as chemotherapeutic agents for advanced NSCLC.

In conclusion, we observed that overexpression of pAkt, mTOR and p70S6K and loss of PTEN expression occur frequently in NSCLCs. The high prevalence of PTEN loss and its association with aggressive tumor behavior in AC suggest that PTEN inactivation is involved in AC progression and PTEN could be a promising therapeutic target for AC treatment. In addition, a significant association between loss of PTEN expression and poor patient outcome in patients with AC suggests that loss of PTEN expression could serve as a prognostic marker for AC.

\section{Acknowledgment}

This work was supported by a grant from the Kyung Hee University Research Fund in 2009 (KHU-20090578).

\section{References}

1 Travis WD, Brambilia E, Muller-Hermelink HK, Harris CC: Pathology and Genetics: Tumours of the Lung, Pleura, Thymus and Heart. Lyon, IARC Press, 2004.

- Jung KW, Won YJ, Park S, Kong HJ, Sung J, Shin HR, Park EC, Lee JS: Cancer statistics in Korea: incidence, mortality and survival in 2005. J Korean Med Sci 2009;24:9951003 .

-3 Vignot S, Faivre S, Aguirre D, Raymond E: mTOR-targeted therapy of cancer with rapa- mycin derivatives. Ann Oncol 2005; 16:525537.

4 Soria JC, Shepherd FA, Douillard JY, Wolf J, Giaccone G, Crino L, Cappuzzo F, Sharma S, Gross SH, Dimitrijevic S, Di Scala L, Gardner H, Nogova L, Papadimitrakopoulou V: Efficacy of everolimus (RAD001) in patients with advanced NSCLC previously treated with chemotherapy alone or with chemotherapy and EGFR inhibitors. Ann Oncol 2009;20:1674-1681.

\footnotetext{
5 Tabernero J, Rojo F, Calvo E, Burris H, Judson I, Hazell K, Martinelli E, Ramon y Cajal S, Jones S, Vidal L, Shand N, Macarulla T, Ramos FJ, Dimitrijevic S, Zoellner U, Tang P, Stumm M, Lane HA, Lebwohl D, Baselga J: Dose- and schedule-dependent inhibition of the mammalian target of rapamycin pathway with everolimus: a phase I tumor pharmacodynamic study in patients with advanced solid tumors. J Clin Oncol 2008;26: 1603-1610
} 
-6 O’Donnell A, Faivre S, Burris HA 3rd, Rea D, Papadimitrakopoulou V, Shand N, Lane HA, Hazell K, Zoellner U, Kovarik JM, Brock C, Jones S, Raymond E, Judson I: Phase I pharmacokinetic and pharmacodynamic study of the oral mammalian target of rapamycin inhibitor everolimus in patients with advanced solid tumors. J Clin Oncol 2008;26: 1588-1595.

7 Travis WD, Brambilla E, Noguchi M, Nicholson AG, Geisinger KR, Yatabe Y, Beer DG, Powell CA, Riely GJ, Van Schil PE, Garg K, Austin JH, Asamura H, Rusch VW, Hirsch FR, Scagliotti G, Mitsudomi T, Huber RM, Ishikawa Y, Jett J, Sanchez-Cespedes M, Sculier JP, Takahashi T, Tsuboi M, Vansteenkiste J, Wistuba I, Yang PC, Aberle D, Brambilla C, Flieder D, Franklin W, Gazdar A, Gould M, Hasleton P, Henderson D, Johnson B, Johnson D, Kerr K, Kuriyama K, Lee JS, Miller VA, Petersen I, Roggli V, Rosell R, Saijo N, Thunnissen E, Tsao M, Yankelewitz D: International Association for the Study of Lung Cancer/American Thoracic Society/European Respiratory Society International Multidisciplinary Classification of Lung Adenocarcinoma. J Thorac Oncol 2011;6:244-285.

8 Edge SB, Byrd DR, Compton CC, Fritz AG, Greene FL, Trotti A: AJCC Cancer Staging Manual. New York, Springer, 2010.

$\checkmark 9$ Noh WC, Kim YH, Kim MS, Koh JS, Kim HA, Moon NM, Paik NS: Activation of the mTOR signaling pathway in breast cancer and its correlation with the clinicopathologic variables. Breast Cancer Res Treat 2008; 110:477-483.

10 Maehama T, Dixon JE: The tumor suppressor, PTEN/MMAC1, dephosphorylates the lipid second messenger, phosphatidylinositol 3,4,5-trisphosphate. J Biol Chem 1998; 273:13375-13378.

- 11 Weng L, Brown J, Eng C: PTEN induces apoptosis and cell cycle arrest through phosphoinositol-3-kinase/Akt-dependent and -independent pathways. Hum Mol Genet 2001;10:237-242.

-12 Kappes H, Goemann C, Bamberger AM, Loning T, Milde-Langosch K: PTEN expression in breast and endometrial cancer: correlations with steroid hormone receptor status. Pathobiology 2001;69:136-142.

-13 Tang JM, He QY, Guo RX, Chang XJ: Phosphorylated Akt overexpression and loss of PTEN expression in non-small cell lung cancer confers poor prognosis. Lung Cancer 2006;51:181-191.

- 14 Yoo SB, Xu X, Lee HJ, Jheon S, Lee CT, Choe $\mathrm{G}$, Chung JH: Loss of PTEN expression is an independent poor prognostic factor in nonsmall cell lung cancer. Korean J Pathol 2011; 45:329-335.
5 Soria JC, Lee HY, Lee JI, Wang L, Issa JP, Kemp BL, Liu DD, Kurie JM, Mao L, Khuri FR: Lack of PTEN expression in non-small cell lung cancer could be related to promoter methylation. Clin Cancer Res 2002;8:11781184.

16 Perren A, Weng LP, Boag AH, Ziebold U, Thakore K, Dahia PL, Komminoth P, Lees JA, Mulligan LM, Mutter GL, Eng C: Immunohistochemical evidence of loss of PTEN expression in primary ductal adenocarcinomas of the breast. Am J Pathol 1999;155: 1253-1260.

17 Duerr EM, Rollbrocker B, Hayashi Y, Peters N, Meyer-Puttlitz B, Louis DN, Schramm J, Wiestler OD, Parsons R, Eng C, von Deimling A: PTEN mutations in gliomas and glioneuronal tumors. Oncogene 1998;16:22592264.

18 Mutter GL, Lin MC, Fitzgerald JT, Kum JB, Baak JP, Lees JA, Weng LP, Eng C: Altered PTEN expression as a diagnostic marker for the earliest endometrial precancers. J Natl Cancer Inst 2000;92:924-930.

19 Rowinsky EK: Targeting the molecular target of rapamycin (mTOR). Curr Opin Oncol 2004; 16:564-575.

-20 Yu K, Toral-Barza L, Discafani C, Zhang WG, Skotnicki J, Frost P, Gibbons JJ: mTOR, a novel target in breast cancer: the effect of CCI-779, an mTOR inhibitor, in preclinical models of breast cancer. Endocr Relat Cancer 2001;8:249-258.

-21 Neshat MS, Mellinghoff IK, Tran C, Stiles B, Thomas G, Petersen R, Frost P, Gibbons JJ, $\mathrm{Wu} \mathrm{H}$, Sawyers CL: Enhanced sensitivity of PTEN-deficient tumors to inhibition of FRAP/mTOR. Proc Natl Acad Sci USA 2001; 98:10314-10319.

-22 Noh WC, Mondesire WH, Peng J, Jian W, Zhang H, Dong J, Mills GB, Hung MC, Meric-Bernstam F: Determinants of rapamycin sensitivity in breast cancer cells. Clin Cancer Res 2004;10:1013-1023.

23 Kobayashi I, Semba S, Matsuda Y, Kuroda Y, Yokozaki H: Significance of Akt phosphorylation on tumor growth and vascular endothelial growth factor expression in human gastric carcinoma. Pathobiology 2006;73:817.

24 Suzuki Y, Shirai K, Oka K, Mobaraki A, Yoshida Y, Noda SE, Okamoto M, Itoh J, Itoh $\mathrm{H}$ Ishiuchi S, Nakano T: Higher pAkt expression predicts a significant worse prognosis in glioblastomas. J Radiat Res (Tokyo) 2010;51: 343-348.
25 Shi Y, Chen L, Li J, Lv YL, Sun Q, Wang LX, Jiao SC: Prognostic and predictive values of pERK1/2 and pAkt-1 expression in nonsmall cell lung cancer patients treated with adjuvant chemotherapy. Tumour Biol 2011; 32:381-390

26 Tanaka Y, Terai Y, Tanabe A, Sasaki H, Sekijima T, Fujiwara S, Yamashita Y, Kanemura M, Ueda M, Sugita M, Franklin WA, Ohmichi M: Prognostic effect of epidermal growth factor receptor gene mutations and the aberrant phosphorylation of Akt and ERK in ovarian cancer. Cancer Biol Ther 2011;11:50-57.

27 Wang X, Yi L, Zhu Y, Zou J, Hong Y, Zheng $\mathrm{W}$ : AKT signaling pathway in invasive ductal carcinoma of the breast: correlation with ER $\alpha, E R \beta$ and HER-2 expression. Tumori 2011;97:185-190.

28 Dobashi Y, Suzuki S, Matsubara H, Kimura M, Endo S, Ooi A: Critical and diverse involvement of Akt/mammalian target of rapamycin signaling in human lung carcinomas. Cancer 2009;115:107-118.

29 Hiramatsu M, Ninomiya H, Inamura K, Nomura K, Takeuchi K, Satoh Y, Okumura S, Nakagawa K, Yamori T, Matsuura M, Morikawa T, Ishikawa Y: Activation status of receptor tyrosine kinase downstream pathways in primary lung adenocarcinoma with reference of KRAS and EGFR mutations. Lung Cancer 2010;70:94-102.

-30 Balsara BR, Pei J, Mitsuuchi Y, Page R, KleinSzanto A, Wang H, Unger M, Testa JR: Frequent activation of AKT in non-small cell lung carcinomas and preneoplastic bronchial lesions. Carcinogenesis 2004;25:20532059.

>31 Wullschleger S, Loewith R, Hall MN: TOR signaling in growth and metabolism. Cell 2006; 124:471-484.

\$2 Thomas GV: mTOR and cancer: reason for dancing at the crossroads? Curr Opin Genet Dev 2006;16:78-84.

33 Buck E, Eyzaguirre A, Brown E, Petti F, McCormack S, Haley JD, Iwata KK, Gibson NW, Griffin G: Rapamycin synergizes with the epidermal growth factor receptor inhibitor erlotinib in non-small-cell lung, pancreatic, colon, and breast tumors. Mol Cancer Ther 2006;5:2676-2684.

34 Boffa DJ, Luan F, Thomas D, Yang H, Sharma VK, Lagman M, Suthanthiran M: Rapamycin inhibits the growth and metastatic progression of non-small cell lung cancer. Clin Cancer Res 2004;10:293-300.

>35 O’Reilly KE, Rojo F, She QB, Solit D, Mills GB, Smith D, Lane H, Hofmann F, Hicklin DJ, Ludwig DL, Baselga J, Rosen N: mTOR inhibition induces upstream receptor tyrosine kinase signaling and activates Akt. Cancer Res 2006;66:1500-1508. 